\section{GW23-e2688 CARDIOVASCULAR RESPONSES AND SUBJECTIVE PERCEIVE DURING DIFFERENT POSITIONS AND RESISTIVE EXERCISES IN HEALTHY ADULTS}

doi:10.1136/heartjnl-2012-302920ab.8
${ }^{1}$ Yuling Wang, ${ }^{2}$ Yafei Wang, ${ }^{1}$ Qi liang, ${ }^{1}$ Yuling Wang. ${ }^{1}$ Department of Rehabilitation Medicine, The First Affiliated Hospital, Sun Yat-sen University; ${ }^{2}$ Department of Rehabilitation Therapy, Zhongshan School of Medicine, Sun Yat-sen University

Objectives Although heart rate variability (HRV) and blood pressure (BP) differences between the transfer of supine and sitting position have long been recognised, limited data are available on the different positions when conduct the resistive exercises. This study aim to examine the HRV, BP, heart rate (HR) and subjective responses for two grade resistive exercises during sitting and supine positions in healthy adults.

Methods We recruited 20 healthy university students (10 males, 10 females; age: $22.0 \pm 2.0$ years) to carry out a sitting and supine position and two intermittent elastic resistive exercise training in a quiet experimental laboratory. The positions and two grade exercises were randomised in order to eliminate the sequence effects. HRV, HR, BP, rating of perceived exertion (RPE) and anxiety were measured after every 5 min task or 5 min rest in sitting or supine position. HRV signal was recorded using the Polar heart rate monitor (Polar Electro, Finland). The R-R interval and spectrum analysis of HRV were performed in fast Fourier transforms (FFT) using a HRV software (Kubios HRV, 2.0, University of Eastern Finland, 2008). The signal of HRV was decomposed into low-frequency (LF; 0.05-0.15 Hz), high-frequency (HF; 0.15-0.5 Hz) components. All data analyses were analysed by SPSS Windows 17.0.

Results We performed a repeated measure ANOVA to examine the variables among four condition tasks. The results indicated the statistically significant differences for LF/HF ratio $\left(F_{(1,19)}=12.184\right.$, $\mathrm{p}=0.002)$, HF $\mathrm{ms}^{2}$ (F $\left.(1,19)=11.290, \mathrm{p}=0.003\right)$, LF n.u. ( $F_{(1,}$ $\left.{ }_{19)}=8.932, p=0.008\right)$, HF n.u. $\left(F_{(1,19)}=11.222, p=0.003\right)$ and HR $\left(F_{(1,19)}=15.818, p=0.001\right)$ between two positions. Moreover, there were significant interactions between positions and graded resistive exercises for LF/HF ratio $\left(F_{(1,19)}=15.386, p=0.001\right), \mathrm{HF}^{2} \mathrm{~ms}^{2} \mathrm{~F}_{(1 \text {, }}$ $\left.{ }_{19}=11.383, p=0.003\right)$, LF n.u. $\left(F_{(1,19)}=9.753, p=0.006\right)$, HF n.u. ( $F$ $(1,19)=16.411, p=0.001)$, and $\operatorname{HR}\left(F_{(1,19)}=14.112, p=0.001\right)$. However, there was no statistical significance for LF/HF ratio $\left(F_{(1}\right.$, $\left.{ }_{19)}=0.941, \mathrm{p}=0.344\right), \mathrm{HF} \mathrm{ms}^{2}\left(\mathrm{~F}_{(1,19)}=3.614, \mathrm{p}=0.073\right), \mathrm{LF}$ n.u. ( $\mathrm{F}$
$(1,19)=1.747, \mathrm{p}=0.202)$, HF n.u. $\left(\mathrm{F}_{(1,19)}=0.448, \mathrm{p}=0.511\right)$ and HR $\left(F_{(1,19)}=2.874, p=0.106\right)$ between the two grades resistance exercises. Paired t-test was also computed to compare the differences among four resistive exercising training and every resting period. There were significant responses for two positions of HR, systolic blood pressure (SBP), diastolic blood pressure (DBP), LF/HF ratio, $\mathrm{HFms}^{2}$, LF n.u. and HF n.u. ( $p<0.05$ ); no significant difference was demonstrated between two grades' resistance training ( $p>0.05)$. The average RPE scores of all conditions were between 7.05 and 10.40. Paired t-test showed that there is a significant difference between RPE at the first resting period during the sitting position (RPE-sR1) and RPE at the third resting period during the sitting position (RPE-SR3) $(p=0.014)$. We didn't find the significant differences between positions as well as the resistive change.

Conclusions This current study results suggested that different positions have an influence on HRV, BP, and HR. The autonomic nerve system shifted the sympathovagal balance toward sympathetic predominance when positions changed from supine to sitting. HRV are more sensitive to assess cardiovascular changes than subjective responses, and it can be used to monitor cardiac autonomic responses. However, further studies are needed, especially in the patients with different diseases. 